\title{
Damage Localization from Projections of Free Vibration Signals
}

\author{
Yi Zhang ${ }^{1}$ and Dionisio Bernal ${ }^{2 *}$
}

\begin{abstract}
An approach for damage localization that operates with free vibration signals in the damaged state is presented. The scheme does not directly point to the damage location but, given a postulated distribution, computes a metric that (for ideal conditions) approaches infinity when the distribution is correct. The approach does not use identification, operates without constraints between the number of sensors and the number of model degrees of freedom and differs from model updating in that only the damage distribution (and not the severity) enters the formulation. Analytical and experimental results are included.
\end{abstract}

Key words: damage localization, free vibration, null space 


\section{Introduction}

This paper presents a scheme for damage localization that operates without identification and which applies whenever it is possible to extract free-vibration signals from the damaged state. The approach applies, therefore, in structures that can be excited using impact, or when free vibration can be synthesized with sufficient accuracy from forced response, typically using the Random Decrement technique [1-6]. The method, designated as the Free Vibration Projection Damage Localization (FvPDL) approach, is derived under the common assumptions of finite dimensionality and linear behavior and operates within theory when the number of sensors, $m$, is no less than the rank of the change in the transfer matrix resulting from damage. In the common case where damage is idealized as changes in the stiffness of the model that replaces the real structure the constraint reduces to $m>\operatorname{Rank}\left(\mathrm{K}_{D}-\mathrm{K}_{R}\right)$; where $\mathrm{K}$ stands for stiffness matrix and the subscripts $\mathrm{D}$ and $\mathrm{R}$ refer to the damaged and the reference states, respectively. This constraint is the same that applies in SDLV and SDDLV [7-8], as well as in the recently introduced S3DL scheme [9].

It's opportune to note that FvPDL does not "point to the damage" but rather provides a metric that is correlated with the "likelihood" that a postulated damage pattern is the correct one; where likelihood has been placed in quotes to indicate that it's been used colloquially and not in the precise sense that it has in probability theory. The metric is a measure of the parallelism between a particular projection of the Fourier transform of the damaged state free vibration and a matrix whose span depends on the postulated damage distribution. The metric is invariant with respect to post-multiplication of the damage distribution by and arbitrary full rank matrix and it thus follows that (for ideal conditions) results are independent of damage severity. The approach is devoid of heuristics and thus guaranteed to succeed when the assumptions used in the derivation are met. The relevant question is, therefore, whether it can operate with sufficient robustness against the limitations imposed by model error and noise in the measurements. To gain some insight into this matter care is taken in the numerical section to incorporate reasonable levels of model error and measurement noise and results from an experimental examination are also included. In its present form the approach is practically restricted to situations where the number of possible damage distributions is manageable.

While there are a number of studies on damage localization were measurements are pre-processed into free decay, these studies do not offer a theoretically grounded connection between the free vibration signals and the damage location but either: 1) used the free decay to perform modal identification, followed by attempts to locate the damage from mode shape changes [10-12], or 2) used heuristics to map the changes in the free decay signals, typically synthesized using the Random Decrement approach, to the damage [13,14]. FvPDL, in contrast, does not use system identification or pattern recognition and, as noted, offers a theoretical link between free vibration signals in the damaged state and the spatial distribution of the damage. Another point of contrast with the techniques of ref.10-14, is the fact that FvPDL is not restricted to specific structural topologies, e.g., beams of frames but applies to any system that has been discretized and modeled. The paper is organized as follows: derivation of the expressions that constitute the approach is presented in Section 2. Sections 3 and 4 describe the approach from an implementation perspective and show results of some numerical experiments. Section 5 shows experimental results obtained when "the damage" is 
a mass perturbation in an aluminum plate and the paper closes in Section 6 with a brief critical review. An appendix containing the expressions needed to implement the approach using a truncated set of modes, as is computationally necessary when the model is large, is also included.

\section{Damage localization from free decay response}

Accepting the typical assumptions of linearity and finite dimensionality and assuming that damage can be treated as a change in the stiffness matrix $\Delta \mathbf{K}$ the response to an arbitrary initial condition in the damaged state can be written as

$$
\mathbf{M} \ddot{\mathbf{x}}_{\mathbf{d}}(\mathbf{t})+\mathbf{C} \dot{\mathbf{x}}_{\mathbf{d}}(\mathbf{t})+(\mathbf{K}-\Delta \mathbf{K}) \mathbf{x}_{\mathbf{d}}(\mathbf{t})=0 \quad \mathbf{x}_{\mathbf{d}}(0)=\mathbf{x}_{0}, \dot{\mathbf{x}}_{\mathbf{d}}(0)=\dot{\mathbf{x}}_{0}
$$

From eq.1 one can write

$$
\mathbf{M} \ddot{\mathbf{x}}_{\mathbf{h}}(\mathbf{t})+\mathbf{C} \dot{\mathbf{x}}_{\mathbf{h}}(\mathbf{t})+\mathbf{K} \mathbf{x}_{\mathbf{h}}(\mathbf{t})=0 \quad \mathbf{x}_{\mathbf{h}}(0)=\mathbf{x}_{0}, \dot{\mathbf{x}}_{\mathbf{h}}(0)=\dot{\mathbf{x}}_{0}
$$

and

$$
\mathbf{M} \ddot{\mathbf{x}}_{\mathbf{p}}(\mathbf{t})+\mathbf{C} \dot{\mathbf{x}}_{\mathbf{p}}(\mathbf{t})+\mathbf{K} \mathbf{x}_{\mathbf{p}}(\mathbf{t})=\Delta \mathbf{K} \mathbf{x}_{\mathbf{d}}(\mathbf{t}) \quad \mathbf{x}_{\mathbf{p}}(0)=0, \dot{\mathbf{x}}_{\mathbf{p}}(0)=0
$$

where it is clear that we've taken

$$
\mathbf{x}_{\mathrm{d}}(\mathbf{t})=\mathbf{x}_{\mathbf{h}}(\mathbf{t})+\mathbf{x}_{\mathbf{p}}(\mathbf{t})
$$

It's opportune to note that although we make reference to the initial state, $\mathbf{x}_{0}$, and to the change in stiffness due to damage, $\Delta \mathbf{K}$, these quantities are not used in the final implementation. Solving eqs. 2 and 3 in Laplace and adding the superscript $o$ to indicate measured coordinates one has

$$
\mathbf{x}_{\mathbf{h}}^{\mathbf{o}}(\mathbf{s})=\mathbf{R}(\mathbf{s})\left\{\begin{array}{l}
\mathbf{x}_{0} \\
\dot{\mathbf{x}}_{0}
\end{array}\right\}
$$

and

$$
\mathbf{x}_{\mathbf{p}}{ }^{\mathbf{0}}(\mathbf{s})=\mathbf{T}(\mathbf{s}) \Delta \mathbf{K} \mathbf{x}_{\mathbf{d}}(\mathbf{s})
$$

where

$$
\mathbf{R}(\mathbf{s})=\mathbf{C}_{\mathbf{u}} \cdot \mathbf{G}(\mathbf{s}) \cdot \mathrm{L}(\mathrm{s})
$$

with

$$
\mathbf{L}(\mathbf{s})=\left[\begin{array}{ll}
(\mathbf{M} \cdot \mathbf{s}+\mathbf{C}) & \mathbf{M}
\end{array}\right]
$$

and

$$
\mathrm{T}(\mathbf{s})=\mathbf{C}_{\mathbf{u}} \mathbf{G}(\mathbf{s})
$$


where $\mathbf{C}_{\mathbf{u}} \in R^{m \times n}$ is a matrix that selects the rows that corresponds to sensor locations and $\mathbf{G}(\mathrm{s})=$ $\left(\mathbf{M s}^{2}+\mathbf{C s}+\mathbf{K}\right)^{-1}$ is the model force to displacement transfer matrix. Since the initial state in eq.5 is not a function of the Laplace variable the expression can be evaluated at k-values of $\mathrm{s}$ and stacked. Combining the stacked equation with the definition introduced in eq.4 one has

$$
\left\{\begin{array}{c}
\mathbf{x}_{\mathbf{d}}^{\mathbf{o}}\left(\mathbf{s}_{1}\right) \\
\vdots \\
\mathbf{x}_{\mathbf{d}}^{\mathbf{o}}\left(\mathbf{s}_{\mathbf{k}}\right)
\end{array}\right\}-\left\{\begin{array}{c}
\mathbf{x}_{\mathbf{p}}^{\mathbf{o}}\left(\mathbf{s}_{1}\right) \\
\vdots \\
\mathbf{x}_{\mathbf{p}}^{\mathbf{o}}\left(\mathbf{s}_{\mathbf{k}}\right)
\end{array}\right\}=\left[\begin{array}{c}
\mathbf{R}\left(\mathbf{s}_{1}\right) \\
\vdots \\
\mathbf{R}\left(\mathbf{s}_{\mathbf{k}}\right)
\end{array}\right]\left\{\begin{array}{c}
\mathbf{x}_{0} \\
\dot{\mathbf{x}}_{0}
\end{array}\right\}
$$

Taking the integer k sufficiently large the matrix on the rhs of eq. 10 becomes tall and is thus guaranteed to have a left null space, which we designate as $\mathbf{Q}_{\mathbf{k}} \in \square^{(\mathbf{m} \cdot \mathbf{k}-2 \mathbf{n}) \mathbf{x}(\mathbf{m} \cdot \mathbf{k})}$. Pre-multiplying eq.10 by $Q_{k}$ gives

$$
\mathbf{Q}_{k}\left\{\begin{array}{c}
\mathbf{x}_{d}^{\mathbf{o}}\left(\mathbf{s}_{1}\right) \\
\vdots \\
\mathbf{x}_{\mathbf{d}}^{\mathbf{o}}\left(\mathbf{s}_{\mathrm{k}}\right)
\end{array}\right\}=\mathbf{Q}_{\mathrm{k}}\left\{\begin{array}{c}
\mathbf{x}_{\mathrm{p}}^{\mathbf{o}}\left(\mathbf{s}_{1}\right) \\
\vdots \\
\mathbf{x}_{\mathrm{p}}^{\mathbf{o}}\left(\mathbf{s}_{\mathrm{k}}\right)
\end{array}\right\}
$$

Writing eq.6 for the k-values of s, pre-multiplying the result by $Q_{k}$ and using the equality in eq. 11 writes

$$
\mathbf{V} \cdot \mathbf{Z}=\mathbf{k}
$$

where

$$
\mathbf{b}=\mathbf{Q}_{\mathbf{k}}\left\{\begin{array}{c}
\mathbf{x}_{\mathbf{d}}{ }^{\mathbf{o}}\left(\mathbf{s}_{1}\right) \\
\mathbf{x}_{\mathbf{d}}{ }^{\mathbf{o}}\left(\mathbf{s}_{2}\right) \\
\vdots \\
\mathbf{x}_{\mathbf{d}}{ }^{o}\left(\mathbf{s}_{\mathbf{k}}\right)
\end{array}\right\}, \quad \mathbf{V}=\mathbf{Q}_{\mathbf{k}}\left(\begin{array}{cccc}
\mathrm{T}\left(\mathbf{s}_{1}\right) \Delta \mathbf{K} & 0 & 0 & 0 \\
0 & \mathrm{~T}\left(\mathbf{s}_{2}\right) \Delta \mathbf{K} & 0 & 0 \\
0 & 0 & \ddots & 0 \\
0 & 0 & 0 & \mathrm{~T}\left(\mathbf{s}_{\mathbf{k}}\right) \Delta \mathbf{K}
\end{array}\right) \mathbf{Z}=\left\{\begin{array}{c}
\mathrm{x}_{\mathbf{d}}\left(\mathbf{s}_{1}\right) \\
\mathrm{x}_{\mathbf{d}}\left(\mathbf{s}_{2}\right) \\
\vdots \\
\vdots \\
\mathrm{x}_{\mathbf{d}}\left(\mathbf{s}_{\mathbf{k}}\right)
\end{array}\right\}
$$

Given a sufficient number of s-values eq.12 is an over-constrained system of equations that is consistent, in the sense that $\mathrm{b}$ is in the span of $\mathrm{V}$, when the damage distribution is correct. The residual between the vector $\mathrm{b}$ and the reconstruction from the span of $\mathrm{V}$ is

$$
\rho=\left(\mathbf{I}-\mathbf{V}^{+} \mathbf{V}\right) \mathbf{b}
$$

where the superscript + stands for pseudoinverse. We use the reciprocal of the norm of this vector as a metric to judge postulated distributions, namely 


$$
\gamma=\frac{1}{\|\rho\|}
$$

with high values of $\gamma$ pointing to the correct distribution. Since the number of rows in $V$ is no less than $m \cdot k-2 n$ and the column rank of this matrix is $k \cdot \operatorname{rank}(\Delta K)$, the span is meaningful when

$$
m>\operatorname{ran}(\not \Delta \mathrm{K})+\frac{2 n}{k}
$$

or,

$$
k \geq \frac{2 n}{m-\operatorname{rank}(\Delta \mathrm{K})}
$$

both equations showing that the lower bound for the number of sensors is $\operatorname{rank}(\Delta \mathrm{K})+1$. We note, for clarity, that

$$
\mathbf{Q}_{k}=\left(\operatorname{Null}\left[\begin{array}{c}
\mathbf{R}\left(\mathbf{s}_{1}\right) \\
\vdots \\
\mathbf{R}\left(\mathbf{s}_{k}\right)
\end{array}\right]^{\mathrm{T}}\right)^{\mathrm{T}}
$$

\section{FvPDL when the Available Response is from Broad Band Excitation}

While FvPDL is most attractive in cases where free vibration is directly measurable, it is evident that the scheme can also be used in cases of broad band excitation by synthesizing free decay using the Random Decrement (RD) technique [1]. The RD technique operates by segmenting the response into $\mathrm{N}$ (typically overlapping) windows in such a way that a bias on the initial state at the start of each window is ensured and free decay is obtained by averaging.

\section{Summary of FvPDL}

1. Formulate a model of the system in the reference state.

2. Collect free vibration data at the available sensors or synthesize free decay from long records of response to broad band excitation using the random decrement technique.

3. Obtain the Fourier transform of the signals and decide on a bandwidth where the energy is significant (so the signal to noise ratio is adequate).

4. Decide on a set of specific frequencies in the bandwidth of the previous step.

5. Stack the Fourier transform at the selected frequencies in a single column to form the vector on the extreme left of eq.10. Note that the operation we refer to here is the integral Fourier transform, not the periodic FFT.

6. Formulate the matrix $\mathrm{R}$ of eq.7 for each of the selected frequencies and compute the matrix $\mathrm{Q}$ from eq.20 (the subscript $\mathrm{k}$ refers to the number of frequencies used). 
7. Compute the vector $\mathrm{b}$ using eq. 13

8. Postulate a damage scenario. This is typically done by treating the damage as a smeared reduction of some characteristic stiffness parameter over a finite region: $\Delta K$ is the difference between the stiffness matrix of the model in the reference and the postulated damaged state. The severity used to compute $\Delta K$ is arbitrary.

9. Formulate the matrix $\mathrm{V}$ of eq.14 (using $\mathrm{T}$ from eq.9, $\mathrm{Q}$ from eq.20 and $\Delta K$ from step 8).

10. Evaluate eq.16 and take the reciprocal as the metric (eq.17).

11. Repeat steps 8 to 10 for all plausible damage distributions.

In the ideal situation the metric of step 10 is large (relative to others) when the damage distribution is correct. Changes in mass can be considered by replacing $\Delta K$ with $\Delta M \cdot s^{2}$.

\section{Computational Scalability}

Formulation of the matrix from where Q is extracted (and the extraction of Q itself) is costly in large models and practicality demands implementation in modal coordinates; the details are summarized in Appendix I.

\section{Numerical Examinations}

We consider a 12-DOFs uniform shear beam with a fundamental period of 1.58 secs, $3 \%$ damping in every mode and acceleration measurements at coordinates $\{2,4 \ldots 12\}$. Model error is introduced (in each simulation) by taking the stiffness of each spring as the true value multiplied by scalars selected from a uniform distribution with lower and upper limits of 0.95 and 1.05. Masses are analogously perturbed using limits of 0.98 and 1.02 and the damping (in each mode) is taken as the true value plus a perturbation, also from a uniform distribution, with limits of $-1 \%$ and $1 \%$. The measured signals are contaminated with independent Gaussian noise having a NSR of $2 \%$ and two damage scenarios are considered: a) loss of $20 \%$ stiffness in spring \#1 and b) loss of $20 \%$ stiffness in spring \#5. The initial conditions do not enter the predictions but they do determine the response and here we've taken them as an idealization of what would result from a hammer impact at mass \#3.

The amplitude of the Fourier transform of the signals (in one simulation) is depicted in fig.1. Inspection shows that there is non-negligible energy in the full bandwidth and we thus opted to take $\omega=2,4,6 \ldots 60 \mathrm{rad} / \mathrm{sec}$. The mean of 50 simulations, where the metric is normalized to a maximum of unity each time, is depicted in fig. 2 for the two scenarios considered. As can be seen, the damaged is correctly localized in both cases, even though the contrast is significantly better when the loss of stiffness is on the first spring. 
Fig.1

Fig.2

\section{$\underline{\text { Moderate Non-Linear Behavior }}$}

In the derivation of FvPDL it is assumed that the system behaves linearly during the data collection. While this assumption is often well-approximated, there are instances where the structure (e.g., composite plates) display nonlinearity even at the small deformation amplitudes and it is of some interest to investigate the sensitivity of the approach to these potential deviations. With the restoring forces in the damage state generalized to $\mathbf{f}\left(\mathbf{x}_{\mathrm{d}}\right)$ one can write;

$$
\mathbf{f}\left(\mathbf{x}_{\mathbf{d}}\right)=\mathbf{K} \mathbf{x}-\Delta \mathbf{K}_{\mathbf{d}} \mathbf{x}+\gamma_{\mathbf{o}}
$$

From where it follows that the difference between the nonlinear restoring force and that in the nominal linear model of the reference state is

$$
\mathbf{f}\left(\mathbf{x}_{\mathbf{d}}\right)-\mathbf{K} \mathbf{x}_{\mathbf{d}}=-\Delta \mathbf{K} \mathbf{x}_{\mathbf{d}}+\Upsilon\left(\mathbf{x}_{\mathbf{d}}\right)
$$

The first term on the right hand side of eq. 22 can be viewed as the first tem of a Taylor series expansion and the second gathers all the nonlinearity. If the nonlinear terms in eq.22 are small, in relative terms, the performance of FvPDL, which takes them as zero, should not be significantly affected, indeed, the one suspects that even significant nonlinearity may have a relatively small effect in the span of the effective force since the response spends most of its time away from the extreme values where the nonlinearity is more pronounced. To get some numerical insight and isolate the nonlinear effect we consider a $4-\mathrm{DOF}$ chain system with masses $=0.05$ and stiffness values $=10$ in a consistent set of units with 5\% modal damping. We consider damage to be a $20 \%$ loss of tangent stiffness at the origin in the first spring and consider two cases: a) the spring is linear (so the theory holds precisely) and b) the spring has a softening elastic nonlinear behavior. Fig. 3 shows the force vs. displacement relation traversed in the free vibration test in both cases.

Fig.3

The normalized metric computed for the two cases are depicted in fig. 4 and show, surprisingly at first: that the resolution in the nonlinear case is somewhat better that when the response is linear. This, of course, is not a general result but it does support our qualitatively derived expectation that the effect of mild nonlinearities in the performance of FvPDL should be small. 
Fig.4

\section{Experimental Examination}

The technique is experimentally tested on a $40.6 \mathrm{~cm} \times 55.9 \mathrm{~cm} \times 0.16 \mathrm{~cm}$ rectangular aluminum (AL-2024) plate. Free-free boundary conditions are approximated by hanging the plate from two bungee cords in a way that gravity is parallel to the plane of the plate. The FE grid, the location of the 9 accelerometers measuring response normal to the plate and the location of a 7 gram added mass used to simulate the damage are depicted in fig.5.

Fig.5

A plot of the absolute value of the Fourier transform of the free vibration at all sensors resulting from the hammer impact is depicted in fig.6. As one anticipates, the signal energy is concentrated around specific resonant frequencies and the bandwidth is large. The first non-rigid body mode of the model is around $170 \mathrm{rad} / \mathrm{sec}$ so the peaks that appear bellow this frequency arise from the geometric stiffness introduced by gravity in the hung configuration.

Fig.6.

Since the damage is a mass perturbation one anticipates that the interrogation should be carried at frequencies where the inertia of the added mass is significant. In selecting these frequencies it is necessary to keep in mind, however, that the accuracy of the FE deteriorates as the frequencies get higher and that the issue is compounded in this case by the fact that while the actual plate is warped the model is a flat plate. With these considerations, and in view of the results depicted in fig.6, we decided on a bandwidth between 450 and $800 \mathrm{rad} / \mathrm{sec}$ and took 20 frequencies equally spaced in it. We performed the analysis using the expressions in Appendix I with a modal space given by the first $10^{\text {th }}$ flexible modes plus the 3 rigid body modes. The highest frequency in the modes selected is $894 \mathrm{rad} / \mathrm{sec}$. The results depicted in fig.7 are a mixed bag. On the positive side the actual mass perturbation is reasonably close to the location of the highest metric but on the other the contrast and resolution of the results are poor; reflection about the middle of the plate in both the $x-x$ and $y-y$ directions showing also high values of the metric. One would expect ambiguity to decrease if the bandwidth is expanded and more modes are added but a pilot examination did not succeed in getting stable results when modes with frequencies above $900 \mathrm{rad} / \mathrm{sec}$ were included in the analysis. As noted previously, this is likely because model error due to unconsidered plate curvature is too large in these frequencies relative to the changes induced by the small mass perturbation. 


\section{Conclusion}

FvPDL is a theoretically grounded scheme for interrogating postulated damage patterns with respect to correctness. The approach operates with a model of the system in the reference condition and a vectorized version of the Fourier transform of free vibration in the damaged state. The scheme does not require that system identification be carried out and does not impose constraints relating the number of DOF in the model and the number of measurements. Since damage severity does not enter the theoretical formulation the approach is a pure localization scheme. Key in the approach is the computation of the null space of the vectorized Fourier transform at a selected set of frequencies for (any possible) initial conditions in the reference state. In the present paper this null space is computed using the matrices of the reference model but a data-driven extraction using free vibration recorded in the reference state is theoretically feasible. Investigation of the merit of this data-driven alternative appears worthwhile.

\section{Acknowledgement}

This research was partly carried out during the stay of the first author at Northeastern University in Boston where he was hosted by the Structural Dynamics and Identification Lab. The support of the National Science Foundation of China under grant No. 11272235 and the China Scholarship Council during this stay is gratefully acknowledged.

\section{Appendix I - Modal Implementation of FvPDL}

Let $\Phi$ be whatever set of modes the user selects to carry out the analysis, contiguous or not, and let their number be $n$, we designate the rows of the modes $\Phi$ associated with sensor coordinate $\Phi^{o}$. In standard fashion, accepting the classically damped model and mass normalization one has the following mapping

$$
\begin{aligned}
& M \rightarrow I_{n \times n} \\
& C \rightarrow \operatorname{diag}\left(2 \omega_{j} \xi_{j}\right) \\
& K \rightarrow \operatorname{diag}\left(\omega_{j}^{2}\right)
\end{aligned}
$$

The reader can confirm that in addition to the above mapping, the matrix $R$ in eq. 7 changes to $R_{M M}$ which is given by

$$
R_{M M}=\tilde{\Phi}^{o} R Z
$$

where

$$
Z=\left[\begin{array}{cc}
\tilde{\Phi}^{o} & 0 \\
0 & \tilde{\Phi}^{o}
\end{array}\right]
$$


and that the coordinate selector $\mathrm{C}_{\mathrm{u}}$ is eliminated, as the mapping to sensor coordinates is realized in the selection of the mode shape coordintes retained in $\tilde{\Phi}^{o}$. The other computational block of FvPDL is the formulation of the matrix $\mathrm{V}$ of eq.14 and in this case examination shows that the the appropriate replacement is

$$
C_{u} G(s) \Delta K \rightarrow \Phi^{o} G \& \Phi^{T} \Delta K
$$

where is worth noting that in the most right appearance of the truncated modes all the coordinates are retained. Needless to say, in the above computations the transfer matrices can be computed from the reduced size matrices of eq.23 or, if desired (more efficiently) as

$$
G(s)=\sum_{n} \frac{\phi_{j} \phi_{j}^{T}}{s^{2}+2 \omega_{j} \xi_{j} s+\omega_{j}^{2}}
$$

Inspection shows that in the modal implementation the matrix $\mathrm{R}_{\mathrm{MM}}$ has $2 \mathrm{n}$ columns so the aggregate matrix from where $\mathrm{Q}$ is extracted becomes tall when the number of frequencies exceeds $2 \mathrm{n}$ divided by the number of sensors.

\section{References}

[1] H.A. Cole, On-the-line analysis of random vibrations, AIAA Paper, (1968).

[2] H.A. Cole, On-line failure detection and damping measurement of aerospace structures by random decrement signatures, National Aeronautics and Space Administration, 1973.

[3] S.R. Ibrahim, Random decrement technique for modal identification of structures, Journal of Spacecraft and Rockets, 14 (1977) 696-700.

[4] J. Vandiver, A. Dunwoody, R. Campbell, M. Cook, A mathematical basis for the random decrement vibration signature analysis technique, Journal of Mechanical Design, 104 (1982) 307-313.

[5] Y. Zhang, L. J. Wu, and H. W. Song, Triggering expectation and residual excitation of distributed random decrement technique, Journal of Sound and Vibration 340 (2015): 368-382.

[6] Y. Zhang, H.W. Song, Non-overlapped random decrement technique for parameter identification in operational modal analysis, Jounal of Sound and Vibration, 366 (2016) 528-543.

[7] D. Bernal, Damage localization from the null space of changes in the transfer matrix, AIAA journal, 45 (2007) 374-381.

[8] D. Bernal, Flexibility-based damage localization from stochastic realization results, Journal of Engineering Mechanics, 132 (2006) 651-658.

[9] D. Bernal, A. Kunwar, Steady State Shift Damage Localization, Meccanica, 51(11), (2016), 2861-2871. 
[10] R. Morsy, H. Marzouk, X. Gu, A. Elshafey, Use of the random decrement technique for nondestructive detection of damage to beams, Materials and Structures, 49(2016) 4719-4727.

[11] A. Elshafey, H. Marzouk, X. Gu, M. Haddara, R. Morsy, Use of fiber Bragg grating array and random decrement for damage detection in steel beam, Eng Struct, 106 (2016) 348-354.

[12] G. Bernagozzi, L. Landi, P.P. Diotallevi, On the Output-Only Vibration-Based Damage Detection of Frame Structures, in: Structural Health Monitoring, Damage Detection \& Mechatronics, Volume 7, Springer, 2016, pp. 23-33.

[13] H.C.H. Li, M. Weis, I. Herszberg, A.P. Mouritz, Damage detection in a fibre reinforced composite beam using random decrement signatures, Compos Struct, 66 (2004) 159-167.

[14] O.V. Shiryayev, J.C. Slater, Detection of fatigue cracks using random decrement signatures, Structural Health Monitoring, 9 (2010) 347-360. 
Figure1

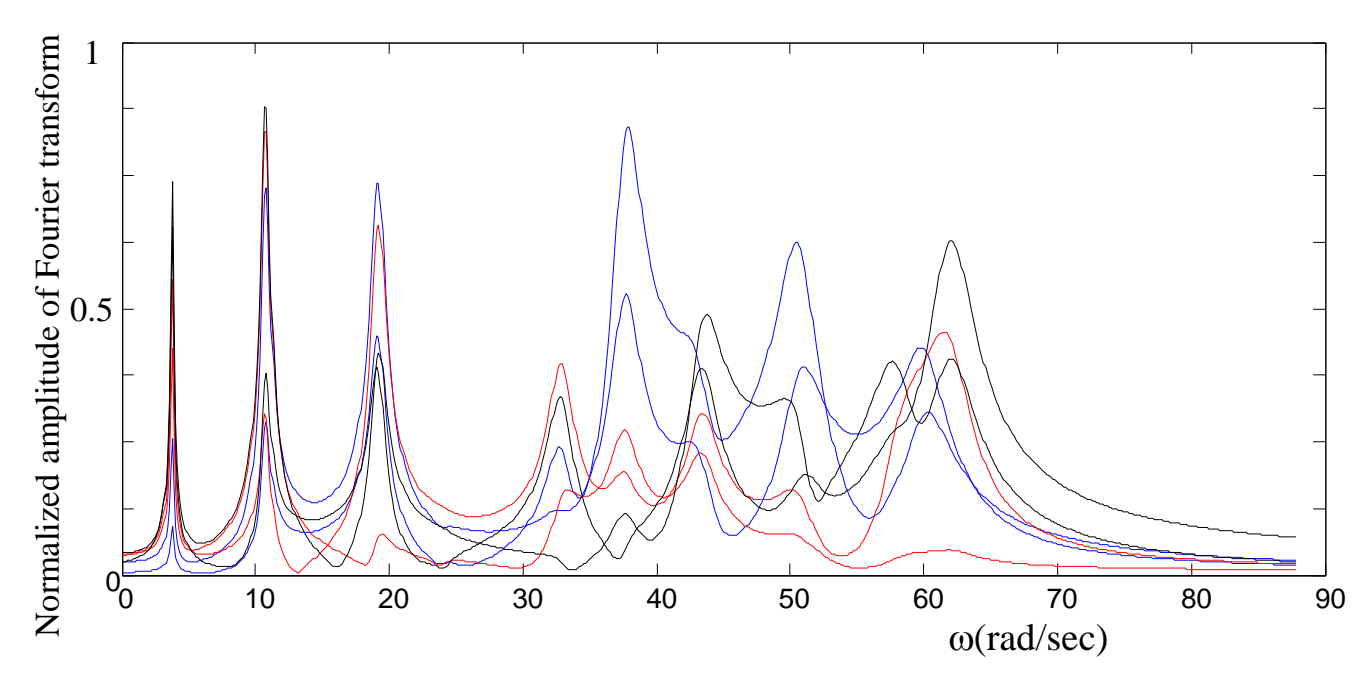

-
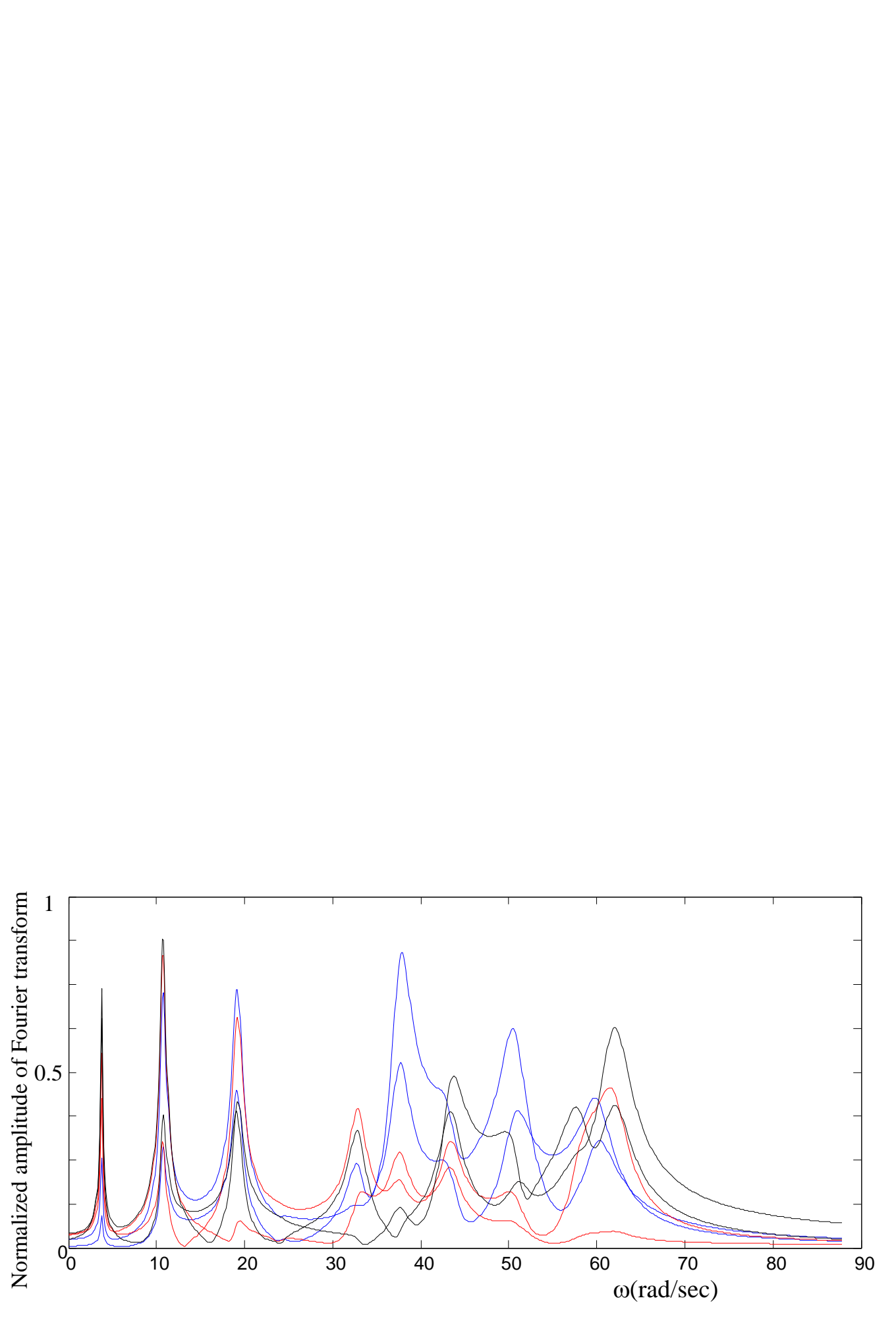

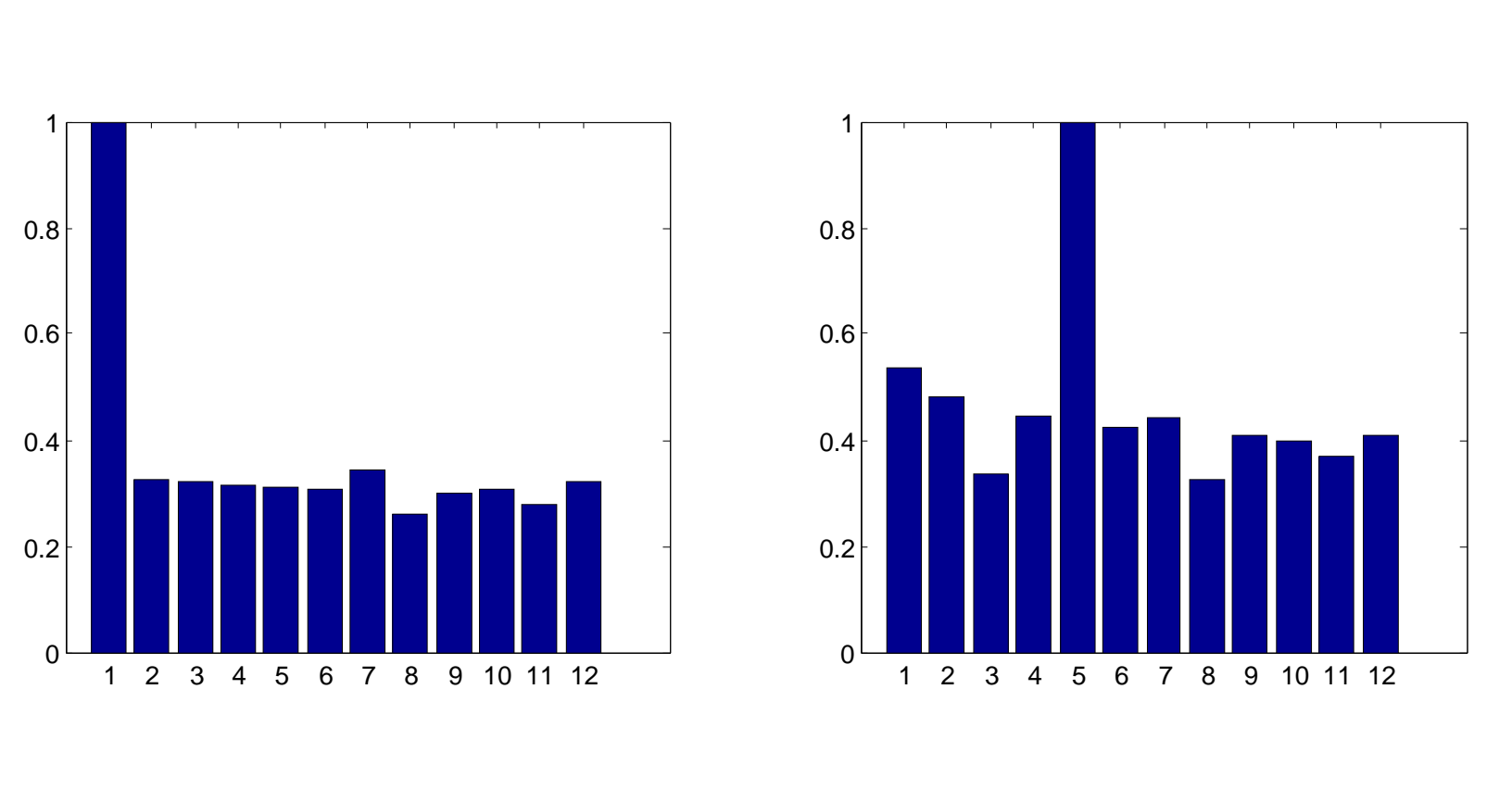

Figure2
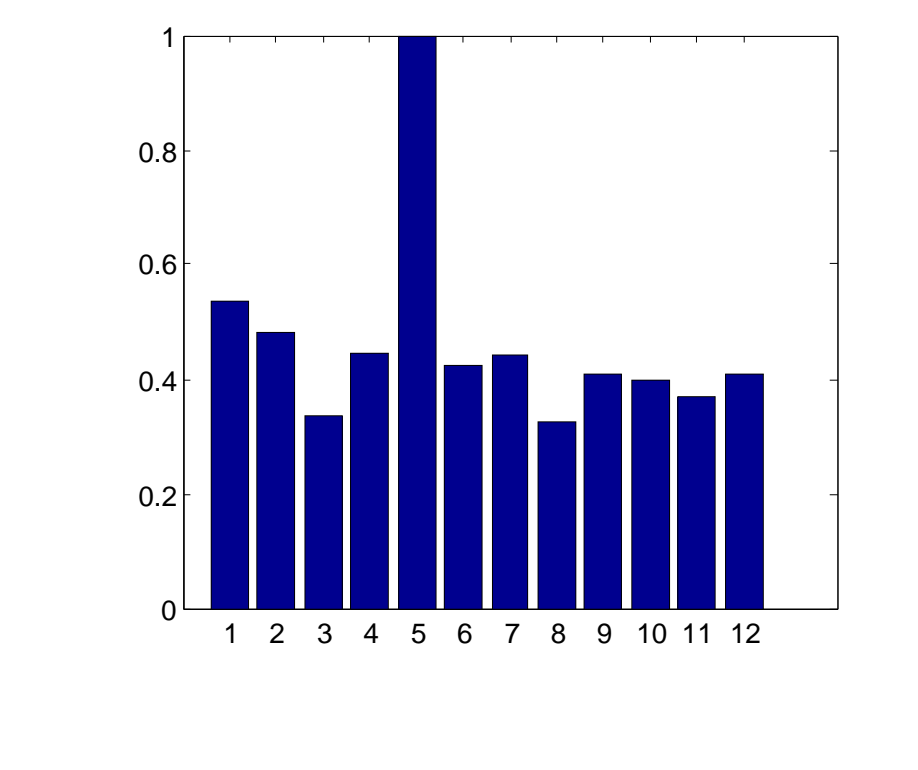

\section{gure2}

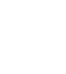

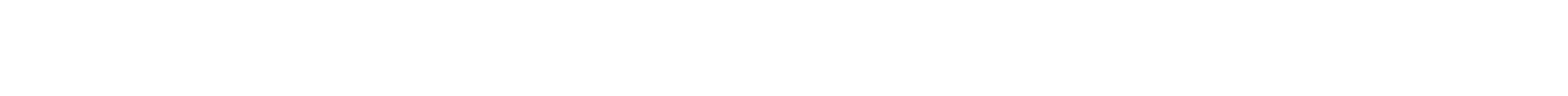

d
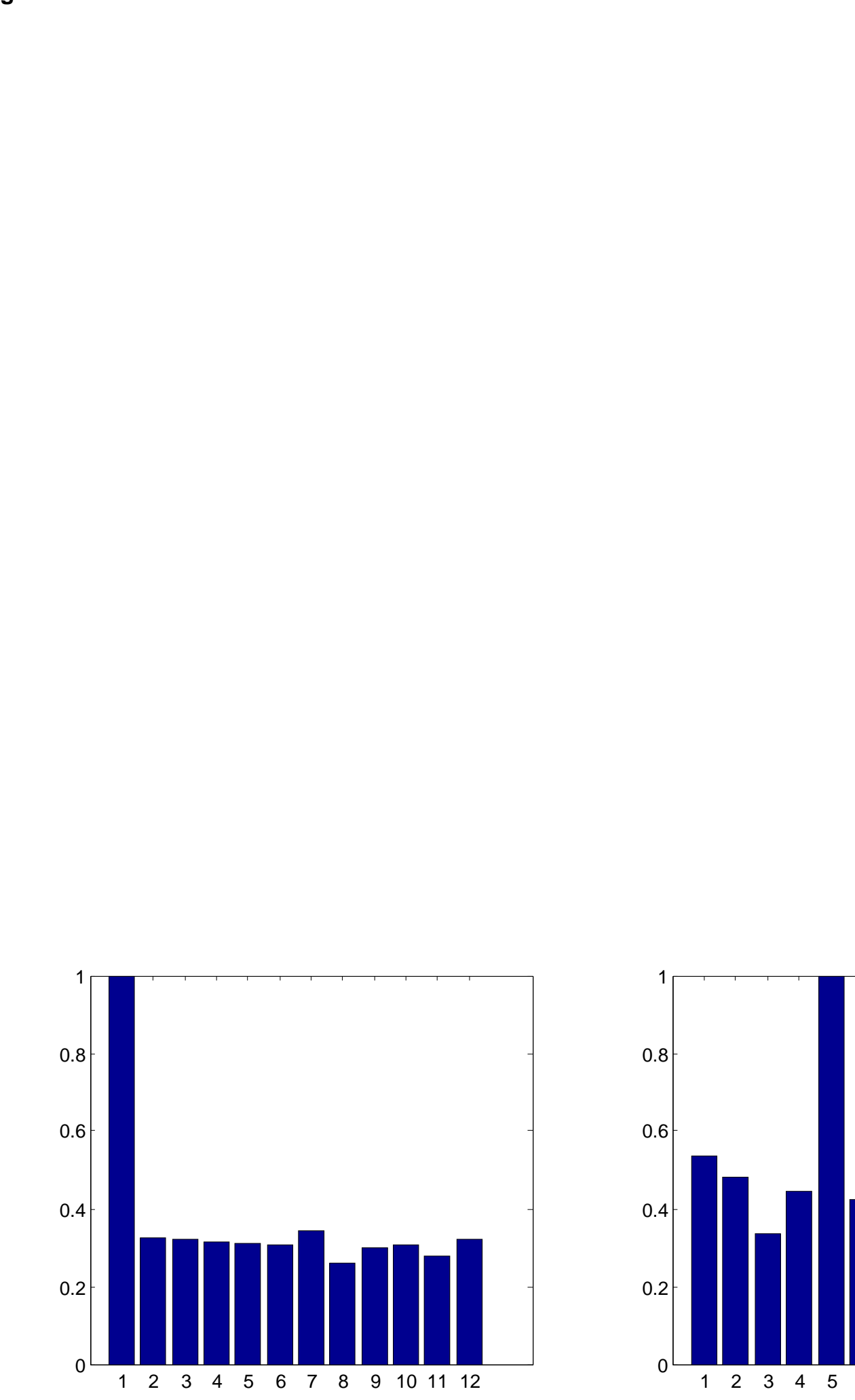
(3) 

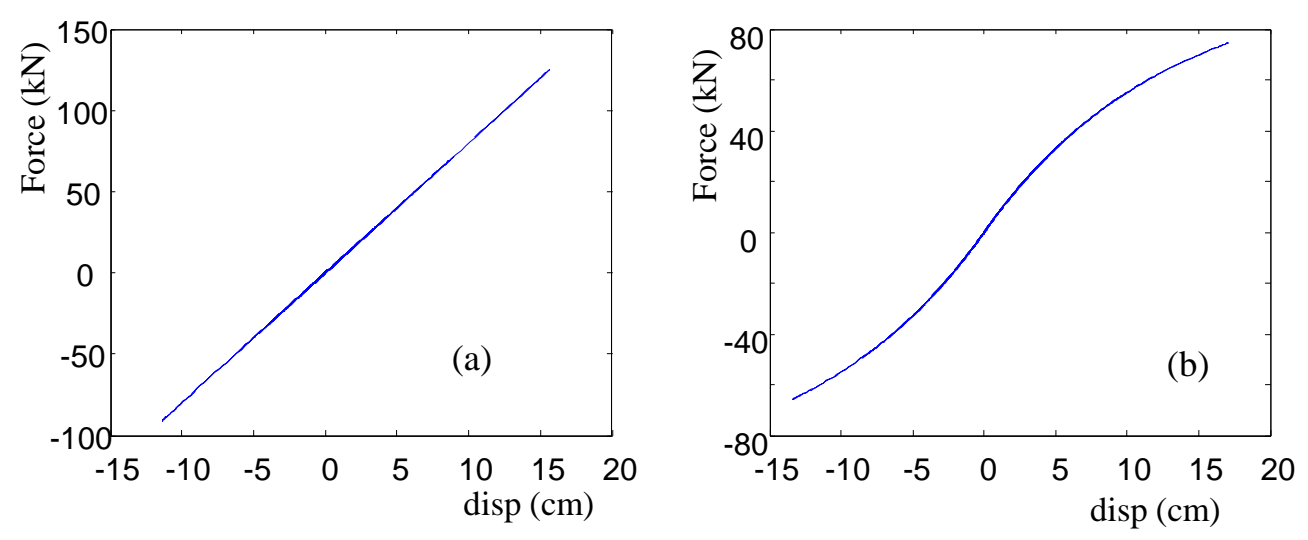

Figure3 

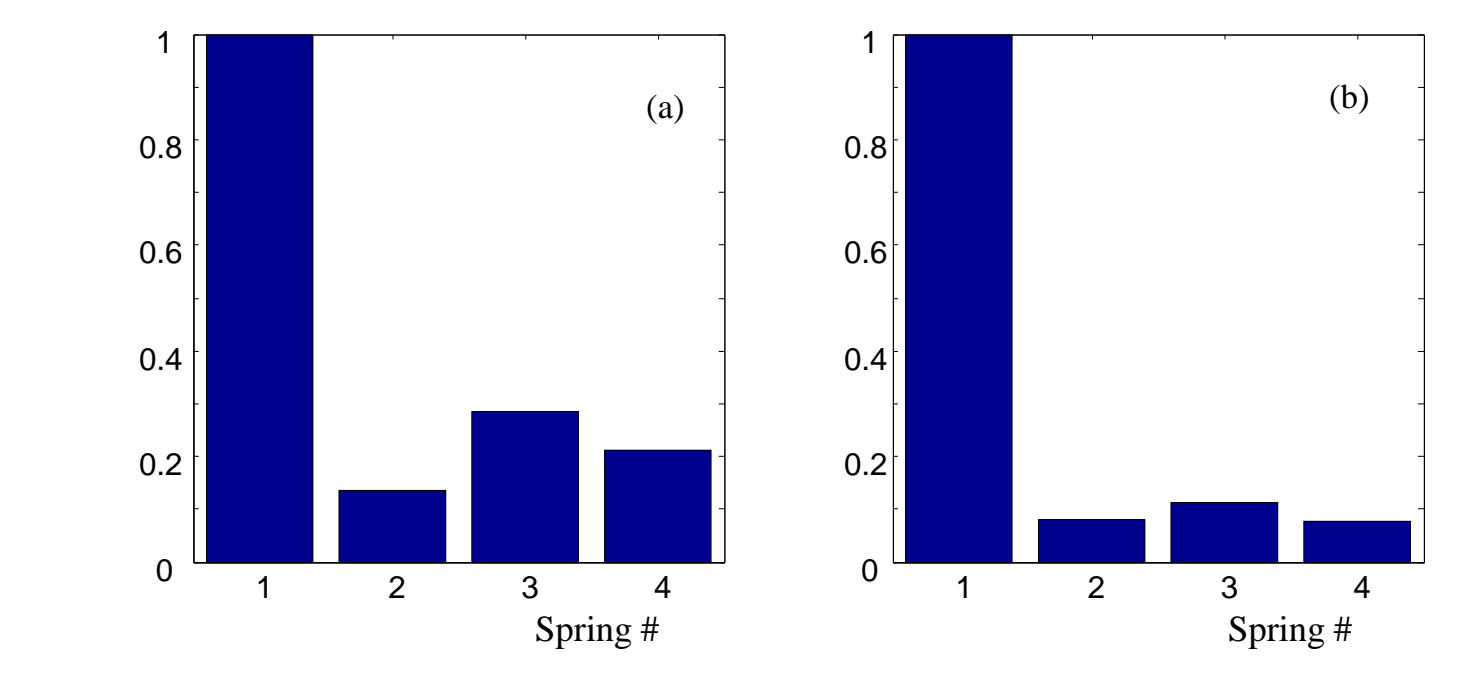

Figure4

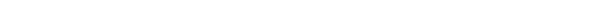

Sping

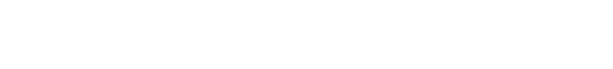


Figure6

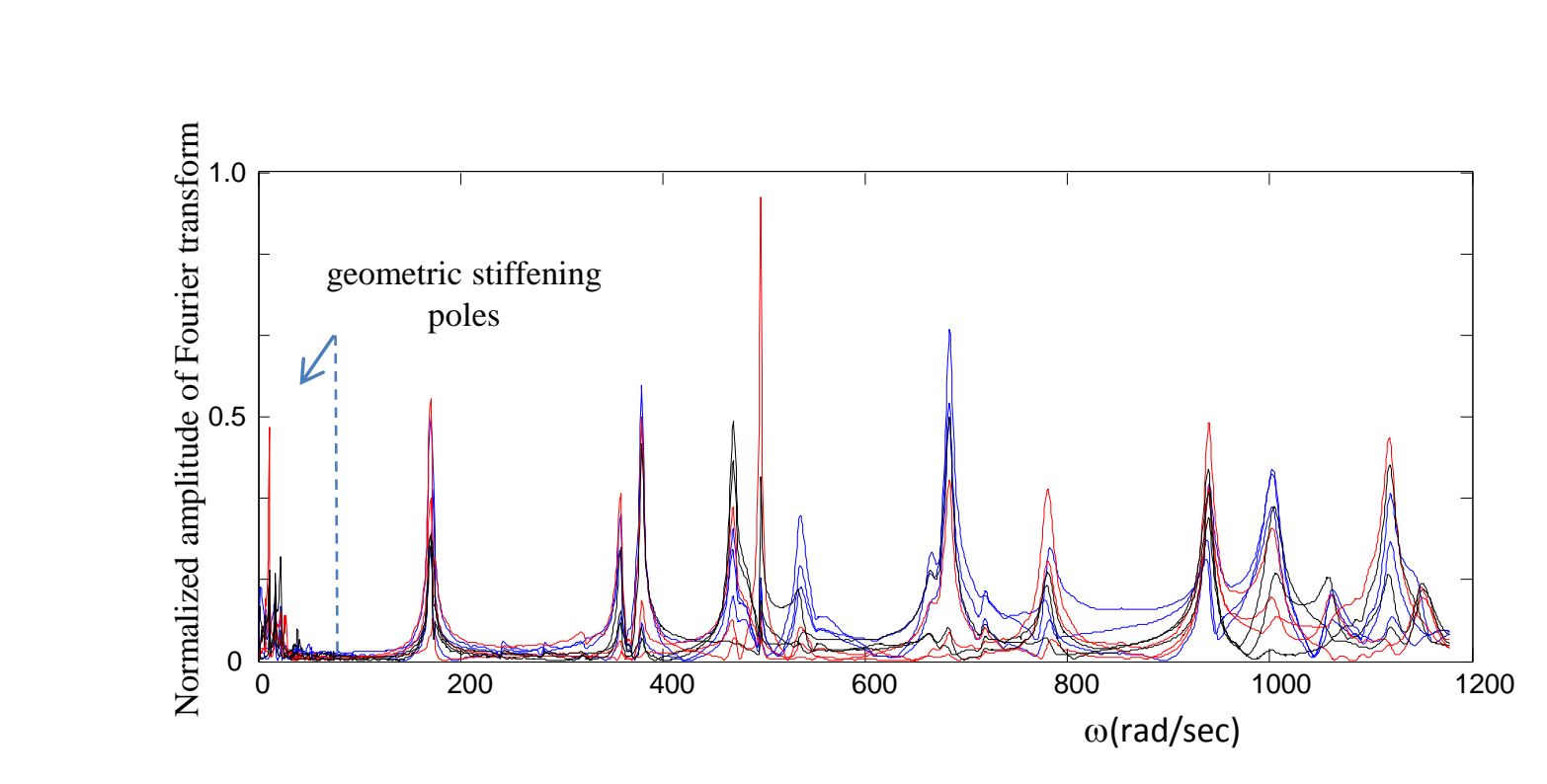

200

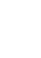

\section{Figure}

'

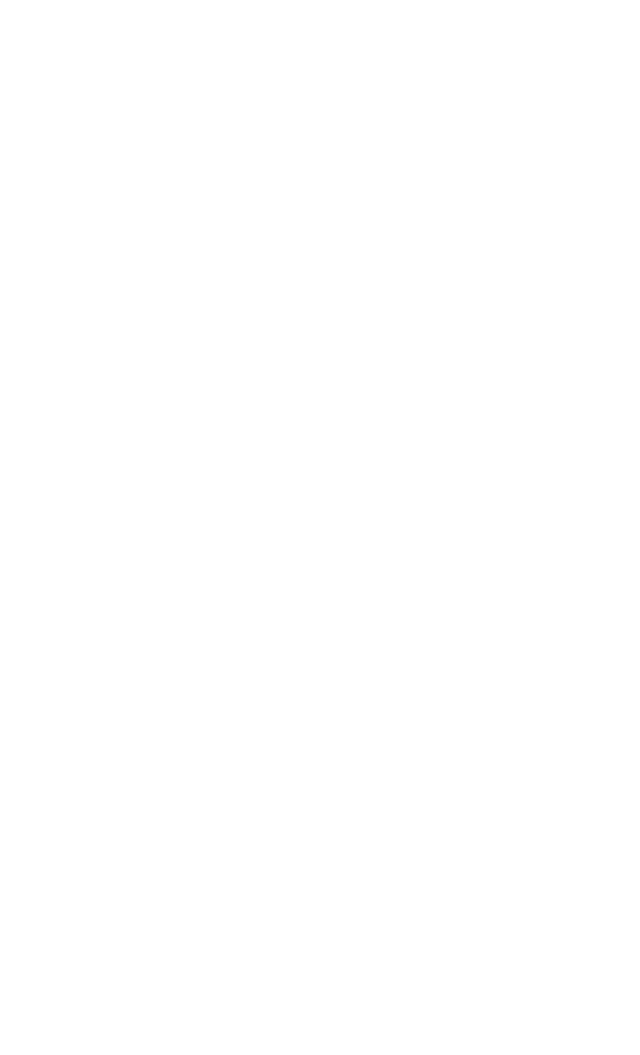




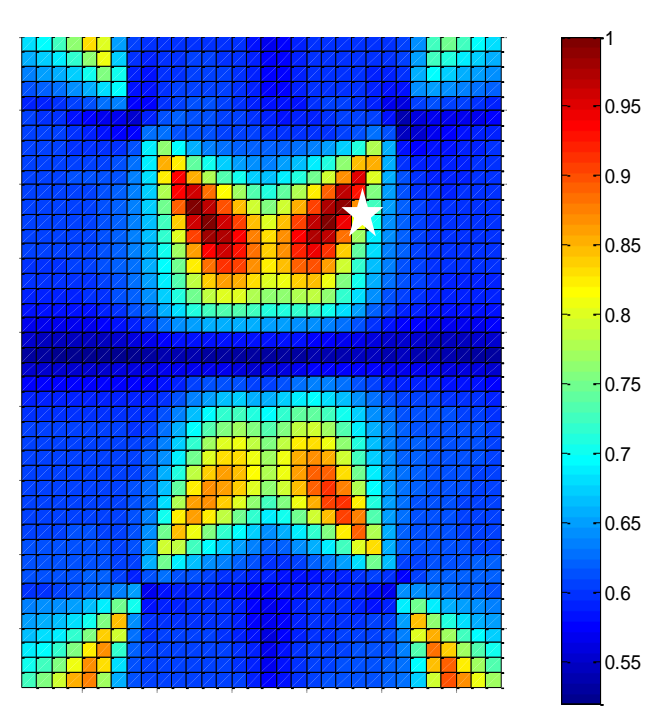

Figure7

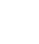

.

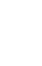


Fig.1 Normalized amplitude of the Fourier transform of the free vibration signal at all measured coordinates (in one simulation)

Fig.2 Metric of eq.17 normalized to maximum of unity (mean of 50 simulations) a) damage on spring \#1 b) damage on spring \#5 (model error and signal noise as described in the text)

Fig.3 Force in the first level spring vs displacement (a) elastic response (b) nonlinear elastic response

Fig.4 Metric of eq.17 normalized to maximum of unity a) linear spring \#1 b) nonlinear spring.

Fig.5 Finite element grid and location of accelerometers (each element is $0.5 \times 0.5$ in with 1 in $=2.54 \mathrm{~cm})$

Fig.6 Absolute value of the Fourier transform at all sensors in the plate.

Fig.7 Predictions of FvPDL on the location of a 7 gram mass perturbation in a $1 \mathrm{~kg}$ plate (star is at the location of the added mass). 